\title{
Lens Iris Diaphragm Retropulsion Syndrome: incidence, risk factors and management. A prospective study.
}

Gabriel Hallali ( $\sim$ GABRIELHALLALI@GMAIL.COM )

Centre Hospitalier Intercommunal de Créteil: Centre Hospitalier Intercommunal de Creteil https://orcid.org/0000-0003-1947-5818

Tristan AUBERT

Centre Hospitalier Intercommunal de Créteil: Centre Hospitalier Intercommunal de Creteil

Eric Souied

Centre Hospitalier Intercommunal de Créteil: Centre Hospitalier Intercommunal de Creteil

Agnès GLACET-BERNARD

Centre Hospitalier Intercommunal de Créteil: Centre Hospitalier Intercommunal de Creteil

\section{Research Article}

Keywords:

Posted Date: February 22nd, 2022

DOI: https://doi.org/10.21203/rs.3.rs-1284339/v1

License: (a) (i) This work is licensed under a Creative Commons Attribution 4.0 International License. Read Full License 


\section{Abstract}

Purpose: The lens-iris diaphragm retropulsion syndrome (LIDRS) corresponds to a brutal deepening of the anterior chamber during phacoemulsification. LIDRS is painful for the patient and sometimes causes intraoperative complications. This study was designed to assess the preoperative risk factors of LIDRS.

Setting: Monocentric study in the university department of ophthalmology, Intercommunal Hospital Center, Creteil, France.

Design: Prospective open-label observational study.

Methods: Preoperative parameters of consecutive patients who underwent cataract surgery were recorded. LIDRS occurrence was evaluated during surgery and classified into 3 stages. Except for the first patient, a simple and rapid maneuver to separate the iris from the capsule (capsular touch) was performed in all patients with LIDRS.

Results: LIDRS occurred in 101 out of 205 included eyes: mild in 73.2\% (74/101) , marked in 16.8\% $(17 / 101)$ and severe in $9.9 \%(10 / 101)$. LIDRS eyes had a longer axial length $(p<0.01)$ and a thinner lens than the control group $(p<0.01)$. Univariate logistic regression showed that the main predictive factors of LIDRS were long axial length, decrease lens thickness. LIDRS occurred in 83\% (5/6) of eyes with prior vitrectomy. Complications occurred (zonular dehiscence), especially in vitrectomized eyes. The capsular touch allowed in almost all eyes to restore normal chamber depth and to prevent complications.

Conclusions: Myopia and prior vitrectomy were the main risk factors of LIDRS. The capsular touch allowed to resolve LIDRS in almost all eyes. Analysis of preoperative factors can help the surgeon to identify patients at risk and to prevent patient pain and intraoperative complications associated with LIDRS.

\section{What Was Known?}

- The lens-iris diaphragm retropulsion syndrome (LIDRS) corresponds to a brutal deepening of the anterior chamber during phacoemulsification.

- LIDRS is painful for the patient and sometimes results in intraoperative complications.

- Risk factors of this syndrome and its management have not been studied in a prospective study.

\section{WHAT THIS PAPER ADDS}

- Myopia and prior vitrectomy are the main predictors of LIDRS occurrence.

- A simple and rapid maneuver to separate the iris from the capsule (capsular touch) allowed in almost all eyes to restore normal chamber depth and to prevent patient pain and intraoperative complications. 


\section{Introduction}

Progressive advances in phacoemulsification techniques made this surgical procedure extremely reproducible. Nevertheless, unexpected problems may occur, such as a sudden and extreme deepening of the anterior chamber. This problem was first described by Zauberman in $1992^{1}$ and referred to as "lensiris diaphragm retropulsion syndrome" (LIDRS) by Willebrandt in 1994². It corresponds to a reversed pupillary block. In this context, the infusion into the anterior chamber provokes an abnormal deepening of the anterior chamber associated with a stretching of the iris, a pupil dilation concomitant with a posterior displacement of the irido-capsular complex. LIDRS can cause severe discomfort to the patient and can lead to intraoperative complications such as capsular rupture or zonular dehiscence ${ }^{2,4}$. Small cases series reported in the literature showed that LIDRS was more frequent in myopic eyes or vitrectomized eyes $^{3}$. Several solutions have been proposed to release the pupillary block, the most effective being the mechanical separation of the iris from the capsular bag using the tip of the irrigation/aspiration cannula ${ }^{2,4}$. In 2017 Lim \& al. ${ }^{5}$ conducted a retrospective cohort study of 124 eyes that reported a frequency of approximately $20 \%$ of this syndrome. Nevertheless, only patients of Korean origin were included, the analysis was done retrospectively, and the evaluation of the effectiveness of intraoperative management of this complication was not possible.

Therefore, this prospective study was designed to evaluate LIDRS incidence in a homogeneous patient cohort and to assess its risk factors. The secondary aim was to evaluate the effectiveness of the LIDRS management procedure.

\section{Methods}

In this prospective study, consecutive patients who underwent cataract surgery between January 2021 and June 2021 were included. This study was conducted in accordance with the tenets of the Declaration of Helsinki (1964) and French legislation after approval by the ethical committee of the hospital center (approval number: 2021-0902).

In order to obtain a homogeneous patient cohort and ensure the reproducibility of the phacoemulsification procedure, all surgeries were performed by the same skilled surgeon (AGB). Surgery indication was indicated in patients with lens opacification causing significant functional discomfort. Patients underwent routine phacoemulsification surgery without complications. Post-traumatic cataract with iris damage, iris pathology, and eyes with insufficient pupillary dilatation requiring iris hooks were excluded.

Before surgery, all patients underwent a complete ophthalmological examination including the record of the patient's history (previous vitrectomy) and characteristics. The measurement of biometric parameters included: axial length (AL), anterior chamber depth (ACD), corneal diameter (white to white, WTW), mean corneal keratometry, theoretical intraocular lens (IOL) power to reach postoperative emmetropia provided 
by the machine with the SRK/T formula and a constant of 118.9. All examinations were performed using the IOLMaster700 (Carl Zeiss Meditec).

\section{Surgical Procedure}

All surgeries were performed using the "Centurion" system (Alcon Laboratories) and under topical local anesthesia. Dilation was achieved by placing a mydriatic insert (Thea Pharma) under the inferior palpebral conjunctiva. The surgical steps consisted of a $2.2 \mathrm{~mm}$ knife incision at the corneal limbus, a continuous curvilinear capsulorhexis with visual guidance (virtual reality, Callisto (Carl Zeiss Meditec)) under viscoelastic $(15.5 \mathrm{mg} / \mathrm{ml}$ sodium hyaluronate, MediconturVivacy), phacoemulsification using the Divide and Conquer technique, cortex removal with a coaxial irrigation/aspiration tip and posterior foldable IOL implantation.

When a combined surgery was decided for an associated retinal disease, cataract surgery was performed before vitrectomy.

\section{Observation and management of LIDRS.}

The surgeon and his assistant were actively watching for the occurrence of LIDRS during the operative times when the fluid infusion was present in the anterior chamber: phacoemulsification, removal of the residual cortex, and final removal of viscosurgical device after IOL implantation.

The LIDRS was classified into 3 grades: grade 1, moderate, characterized by a deepening of the anterior chamber that quickly resolved after the maneuver described below; grade 2, marked, with a blockage that recurred several times during surgery and resulted in a stretched and floppy iris with loosening of the mydriasis; grade 3, severe, characterized by the occurrence of complications (zonular dehiscence, lens or IOL dislocation).

In case of LIDRS occurrence, the "capsular touch"4 maneuver was performed in all patients except the first one. It consisted of a moderate pressure on the anterior capsule using the irrigation/aspiration tip to achieve a mechanical separation of the iris from the capsular plane and release the reverse pupillary block. This separation could also be done using the micromanipulator through the counter incision during the phacoemulsification phase.

This maneuver had to be performed as soon as possible after the beginning of the deepening. Therefore, to control the infusion of the anterior chamber and prevent the sudden and extreme deepening of the anterior chamber, the infusion line was systematically pinched in all patients when entering the irrigation/aspiration tip in the anterior chamber. It permitted to decrease the flow in the anterior chamber and to have sufficient time to make the capsular touch if the LIDR occurred.

\section{Statistical Analysis:}


All statistical analyses were performed with SPSS software (IBM, version 24). Patients with LIDRS were identified as the LIDRS group, and patients without LIDRS were identified as the control group. Statistical tests were two-tailed with a significance level of 0.05 . In case of missing data, calculations were made, taking into account only the available data without imputation.

\section{Results}

This prospective study consisted of 205 eyes of 148 patients, including 140 female (68\%), with a mean age of 74.9 years, (range, 36 to 95 ). The same proportion of right and left eyes was included (108 right eyes vs 97 left eyes). Planned combined surgery was performed in 16 eyes (8\%) for epiretinal membrane peeling or macular hole surgery. Six eyes experienced prior vitrectomy for macular hole (4 eyes), epiretinal membrane (1 eye), retinal detachment (1 eye).

LIDRS occurred in 101 eyes (49\%): grade I in 74 eyes, grade II in 17 eyes, and grade III in 10 eyes (Table 1). Biometric measurements and patients' characteristics are shown in Table 2, in the LIDRS group and the control group. There was a statistically significant difference in age, axial length, lens thickness, and theoretical IOL power to reach postoperative emmetropia. LIDRS occurred more often in younger patients, eyes with long axial length (myopia), low lens thickness, and low theoretical IOL power.

Table 1

Incidence and grade of lens-iris diaphragm retropulsion syndrome in the series of 205 operated eyes

\begin{tabular}{|c|c|c|c|c|c|c|}
\hline & $\begin{array}{l}\text { Number of } \\
\text { patients (\%) }\end{array}$ & Age & $\begin{array}{l}\text { Intraoperative } \\
\text { complication } \\
(\%)\end{array}$ & $\begin{array}{l}\text { Axial } \\
\text { length }\end{array}$ & $\begin{array}{l}\text { Anterior } \\
\text { chamber depth }\end{array}$ & IOLemm \\
\hline $\begin{array}{l}\text { LIDRS } \\
\text { Grade } 1\end{array}$ & $74(36)$ & $\begin{array}{l}73.9 \pm \\
7.12\end{array}$ & $3(4)$ & $\begin{array}{l}24.12 \pm \\
2.03\end{array}$ & $3.24 \pm 0.30$ & $\begin{array}{l}19,96 \pm \\
6.04\end{array}$ \\
\hline $\begin{array}{l}\text { LIDRS } \\
\text { Grade } 2\end{array}$ & $17(8)$ & $\begin{array}{l}66.1 \pm \\
12.89\end{array}$ & 3 (18) & $\begin{array}{l}25.79 \pm \\
3.28\end{array}$ & $3.58 \pm 0.70$ & $\begin{array}{l}14.41 \pm \\
7.09\end{array}$ \\
\hline $\begin{array}{l}\text { LIDRS } \\
\text { Grade } 3\end{array}$ & $10(5)$ & $\begin{array}{l}71.5 \\
\pm 7.75\end{array}$ & 7 (70) & $\begin{array}{l}25.11 \pm \\
2.31\end{array}$ & $3.29 \pm 0.49$ & $\begin{array}{l}15.30 \pm \\
5.70\end{array}$ \\
\hline
\end{tabular}


Table 2

Comparison of clinical factors between eyes with lens-iris diaphragm retropulsion syndrome and control eyes

\begin{tabular}{|c|c|c|c|}
\hline & LIDRS group $(n=101)$ & Control group $(n=104)$ & $P$ value \\
\hline Mean age & $72.36 \pm 8.81$ & $77.40 \pm 7.07$ & $<0.01^{*}$ \\
\hline Right eyes (\%) & $49(48.51)$ & $55(52.88)$ & 0.39 \\
\hline Prior vitrectomy (\%) & $5(4.95)$ & $1(0.96)$ & \\
\hline Axial length (mm) & $24.50 \pm 2.38$ & $23.46 \pm 3.07$ & $<0.01^{*}$ \\
\hline Anterior Chamber Depth (mm) & $3.31 \pm 0.68$ & $3.05 \pm 0.39$ & 0.33 \\
\hline Lens Thickness (mm) & $4.63 \pm 1.15$ & $4.82 \pm 0.81$ & $<0.01 *$ \\
\hline White to White $(\mathrm{mm})$ & $1.20 \pm 0.05$ & $1.29 \pm 0.04$ & 0.32 \\
\hline Mean Keratometry (diopter) & $43.85 \pm 1.44$ & $43.94 \pm 1.51$ & 0.66 \\
\hline IOLemm (diopter) & $18.56 \pm 4.12$ & $21.19 \pm 2.29$ & $<0.01^{*}$ \\
\hline \multicolumn{4}{|c|}{$\begin{array}{l}\text { Values are presented as mean value } \pm \text { standard deviation or as number }(\%) . \text { LIDRS }=\text { lens iris } \\
\text { diaphragm retropulsion syndrome. } *=\text { significative test. IOLemm : theoretical intraocular lens }(\text { IOL }) \\
\text { power to reach postoperative emmetropia }\end{array}$} \\
\hline
\end{tabular}

Eighty-three percent of eyes with prior vitrectomy had operative LIDRS. Among the 16 eyes that underwent combined surgery, 50\% (8/16) experienced LIDRS.

In the LIDRS group, 13 eyes experienced intraoperative complications (Table 1) that occurred during the cortex removal phase or the final removal of viscosurgical device after IOL implantation, which represents $6 \%(13 / 205)$ of all operated eyes, and 13\% (13/101) of LIDRS eyes. These complications never occurred in the control group. The most frequent complication (10 eyes) was iris stretching causing secondary iris atony responsible for intraoperative mydriasis release, hampering the rest of the surgical procedure (cortex removal, IOL implantation). The LIDRS resulted in an anterior capsular tear in one eye. In 2 eyes, zonular dehiscence occurred. Both eyes had prior vitrectomy for macular hole surgery. In the first case, an emmetropic man with a dark iris, the zonular dehiscence was limited and an IOL could be placed in the capsular bag using a capsular tension ring. In the second case, a highly myopic woman with a blue iris (the first patient in whom the maneuver to release the iris block was not performed), the zonular dialysis was almost total resulting in a dislocated IOL that required secondary implantation with an IOL clipped to the iris.

Table 2 shows the result according to LIDRS occurrence. In the cohort, 28 patients had an axial length greater than $25 \mathrm{~mm}$. In this subgroup, the incidence of LIDRS was significantly higher: $67 \%(19 / 28)$ vs $46 \%(82 / 177)$ in the subgroup with axial length less than $25 \mathrm{~mm}$ (Chi 2 test $=4.48, p<0.025)$. Table 3 shows the odds ratios for clinical characteristics associated with LIDRS based on univariable logistic 
regression analysis. Statistically significant correlations with LIDRS were found for age, axial length, lens thickness, and theoretical IOL power to reach postoperative emmetropia.

Table 3

Univariate logistic regression for lens-iris diaphragm retropulsion syndrome risk factors.

\begin{tabular}{|llccc|}
\hline & Regression coefficient & OR & $95 \%$ Cl & p-value \\
\hline Age & -0.0385 & 0.96 & $(0.93 ; 0.99)$ & $0.038^{*}$ \\
\hline Axial length & 0.4432 & 1.56 & $(1.23 ; 1.98)$ & $0.0003^{*}$ \\
\hline Lens Thickness & -1.2963 & 0.27 & $(0.12 ; 0.61)$ & $0.0014^{*}$ \\
\hline IOLemm & -0.0391 & 0.96 & $(0.79 ; 1.16)$ & $0.09^{*}$ \\
\hline IOLemm : theoretical intraocular lens power to reach postoperative emmetropia. * = significative test. \\
\hline
\end{tabular}

In all but the first eye of the series, the management of LIDRS consisted of a moderate pressure on the anterior capsule (capsular touch) using the irrigation/aspiration tip to achieve a mechanical separation of the iris and the capsular plane and release the reverse pupillary block. This procedure allowed to release the LIDRS at least temporarily. This procedure allowed the pathological deepening of the anterior chamber to be stopped, the dilation of the iris to be respected, and the pain of the patient to be reduced. In one eye, the LIDRS occurred numerous times despite the maneuver, and localized zonular dehiscence still occurred. In the case in which the maneuver was not performed (the first patient of the series), extensive zonular dialysis resulted in a complete dislocation of the IOL (described in the complication paragraph above).

\section{Discussion}

In the current study, the first prospective study of a large cohort of Caucasian patients designed to evaluate the occurrence and management of LIDRS, the incidence of LIDRS was $49 \%$ in the total group, $67 \%$ in myopic eyes, and $89 \%$ in vitrectomized eyes. The main risk factors for the occurrence of this syndrome were young age, axial length over $25 \mathrm{~mm}$, and decreased lens thickness.

The incidence of LIDRS in the total cohort was $49 \%$, including $75.2 \%$ of moderate forms, $14.8 \%$ of marked forms, and $9.9 \%$ of severe forms. The incidence was higher than reported in the literature. Lim and coworkers ${ }^{5}$ reported an incidence of $19.4 \%$ including $5.6 \%$ grade $1,10.5 \%$ grade 2 and $3.2 \%$ grade 3 . However, this was a retrospective study where some cases may have been missed. In the current study, the occurrence of LIDRS was actively monitored and included even moderate LIDRS.

The univariate logistic analysis showed that young age was a risk factor for LIDRS. This may be due to a bias since myopic patients had cataract surgery at a younger age. 
Prior vitrectomy is a well-known condition associated with LIDRS. In 1998, in a prospective study of 23 eyes with prior vitrectomy, Diaz Lacalle and coworkers ${ }^{6}$ reported a LIDRS incidence of $26 \%$. The team of $\mathrm{Lim}^{5}$ reported an incidence of $42 \%$ in vitrectomized. In the current study, the incidence was higher, but the series included only 6 eyes with a history of vitrectomy; this discrepancy may be explained by sampling fluctuations, and/or by the occurrence of moderate stage LIDRS not taken into account in the previous studies. Moreover, in this study, the most important complications (zonular dehiscence and dislocation of the IOL) occurred in 2 patients who had previously undergone vitrectomy for macular holes. The more frequent occurrence of LIDRS in vitrectomized eyes might be related to the absence of vitreous support, as suggested by Diaz and coworkers ${ }^{6}$ : the infusion pressure could not be counterbalanced by the vitreous pressure. A second active infusion into the eye has been proposed by some surgeons for cataract surgery on vitrectomized eyes 7,8 : either an infusion into the posterior segment by a 23-gauge pars plana trocar through the sclera or intravitreal injection of BSS at the beginning of the operation. These techniques were not used in this study.

Several techniques have been proposed in the literature for the management of the LIDRS, especially by Cionni and coworkers ${ }^{9}$ who described precisely a technique for the mechanical separation of the iris from the capsule. Some surgeons proposed to change the orientation of the cap of the irrigation/aspiration tip to vertical instead of horizontal. It was also suggested to modify the irrigation pressure or the height of the infusion bottle. Nevertheless, changing these parameters may require some time during which the LIDRS may worsen. The technique of mechanical separation of the iris from the capsule, the capsular touch performed in this study, was easy and rapid and gave an immediate result. To control the infusion of the anterior chamber and to have sufficient time to make the capsular touch if the LIDR occurred, the infusion line was systematically pinched in all patients when entering the irrigation/aspiration tip in the anterior chamber. This procedure permitted us to prevent the sudden and extreme deepening of the anterior chamber, to release the LIDRS when present, and to avoid severe complications in all cases except one in our series. Moreover, it allowed to relieve patient discomfort during surgery and therefore increases intervention security. ${ }^{10}$

Our study was limited by a potential classification bias when assessing the occurrence of LIDRS. LIDRS frequency may have been overestimated because of the special attention paid during surgery to this problem. Furthermore, the presence of capsular exfoliation was not taken into account in the data collection. The presence of this syndrome might favor the occurrence of LIDRS because of associated zonular weakness. ${ }^{1,2}$

In conclusion, LIDRS occurred in $49 \%$ of eyes during cataract surgery especially in myopic and/or vitrectomized eyes, and might be a source of pain and possible intraoperative complications. Therefore, preventing the occurrence of this syndrome is essential to ensure safe surgery. Increased attention from the surgeon is required particularly at the beginning of the cortex removal phase in eyes with a history of vitrectomy or an increased axial length over $25 \mathrm{~mm}$. The capsular touch technique was effective to 
separate the iris from the capsule, releasing the pupillary block, and thus decreasing the patient's discomfort and the risk of complications.

\section{Declarations}

No financial disclosure

No meeting presentation

\section{References}

1. Zauberman H (1992 Aug) Extreme deepening of the anterior chamber during phacoemulsification. Ophthalmic Surg 23(8):555-556

2. Wilbrandt HR, Wilbrandt TH (1994 Jan) Pathogenesis and management of the lens-iris diaphragm retropulsion syndrome during phacoemulsification. J Cataract Refract Surg 20(1):48-53

3. Ghosh S, Best K, Steel DHW (2013 Dec) Lens-iris diaphragm retropulsion syndrome during phacoemulsification in vitrectomized eyes. J Cataract Refract Surg 39(12):1852-1858

4. Glacet-Bernard A, Lasnier M, Karsenty M, Souied E (2019) A small touch that can save your phakos: balancing the pressures between the anterior and posterior chambers. Communication presented. French Society of Ophthalmology, Paris, France

5. Lim DH, Shin DH, Han G, Chung E-S, Chung T-Y (2017 Aug) The Incidence and Risk Factors of Lensiris Diaphragm Retropulsion Syndrome during Phacoemulsification. Korean J Ophthalmol KJO 31(4):313-319

6. Díaz Lacalle V, Orbegozo Gárate FJ, Martinez Alday N, López Garrido JA, Aramberri Agesta J (1998 Jun) Phacoemulsification cataract surgery in vitrectomized eyes. J Cataract Refract Surg 24(6):806809

7. Lyu J, Zhao P Intraoperative Posterior Chamber Irrigation to Enhance Vitreous Cavity Support during Phacoemulsification Cataract Surgery after Vitrectomy.J Ophthalmol. 2020 Feb17;2020:1-6

8. Al Amro SA Managing Challenges of Phacoemulsification in Vitrectomized Eyes: A New Technique.Middle East Afr J Ophthalmol. 2020 Apr29;27(1):53-5

9. Cionni RJ, Barros MG, Osher RH (2004 May) Management of lens-iris diaphragm retropulsion syndrome during phacoemulsification. J Cataract Refract Surg 30(5):953-956

10. Kang YK, Kim MJ, Kim HK, Chun BY (2017) Clinical Analysis of Ocular Parameters Contributing to Intraoperative Pain during Standard Phacoemulsification. J Ophthalmol 2017:9463871 